\title{
PROPOSTA DE MEDIÇÃO DO DESEMPENHO ENERGÉTICO PARA MAPAS DE FLUXO DE VALOR ESTENDIDOS A ENERGIA
}

Fabrício Nicoletti Hedlund (fabricio.hedlund@gmail.com) - Universidade Federal de Santa Catarina

Fernando Antônio Forcellini (fernando.forcellini@ufsc.br) - Universidade Federal de Santa Catarina

Diego Castro Fettermann (d.fettermann@ufsc.br) - Universidade Federal de Santa Catarina

\section{RESUMO}

A melhoria do desempenho energético é fundamental para o desenvolvimento sustentável, em especial no setor industrial, que representa a maior parcela do consumo e espera-se que continue tendo a maior participação do uso final de energia até 2040. Ferramentas que auxiliam a melhoria do desempenho operacional, como o Mapa de Fluxo de Valor, foram estendidas para incorporar variáveis energéticas. Entretanto, existe uma lacuna em relação a forma na qual o desempenho energético é mensurado nestes mapas, inclusive inviabilizando esta informação para benchmarking de forma interna ou externa às organizações. Dessa forma, este estudo tem o propósito de propor um processo de medição do desempenho energético em mapas de fluxo de valor auxiliando a direcionar os esforços do setor industrial, bem como a sua correta utilização para fins de benchmarking por meio de normas e protocolos internacionais, como a ISO 50.001 e o International Performance Measurement and Verification Protocol. A proposta apresentada acrescenta atividades nas etapas de desenvolvimento do Mapa de Fluxo de Valor, contribui para a geração de uma base de conhecimento e suporte na tomada de decisões.

Palavras chave: medição de desempenho; energia; mapa de fluxo de valor; melhoria contínua, desempenho energético 


\section{INTRODUÇÃO}

$\mathrm{O}$ desempenho energético tem um papel importante em direção ao desenvolvimento sustentável. Isto porque a demanda mundial de energia em 2018 aumentou quase o dobro da taxa média de crescimento desde 2010, resultando em um novo recorde de emissões de $\mathrm{CO}_{2}$. Mesmo que o mundo esteja enfrentando uma transição de energia fóssil para renovável, a demanda global de carvão cresceu pelo segundo ano em 2018 (IEA, 2019). Além disso, a eficiência energética, pelo terceiro ano consecutivo, teve sua taxa de melhoria na intensidade global de energia primária decrescida, sendo que a eficiência energética é a maior contribuidora para a redução das emissões de dióxido de carbono. O desempenho energético se relaciona com pelo menos três resultados mensuráveis diferentes, ou seja, consumo, uso e eficiência de energia (ISO, 2011). Portanto, o desempenho energético deve ser aprimorado continuamente para que minimize impactos negativos devido às necessidades de utilização da energia relacionadas às condições econômicas, ambientais e sociais.

O setor industrial destaca-se em direção a sustentabilidade devido a representar a maior parcela do uso final de energia no mundo (LAWRENCE et al., 2019) e por se esperar que ele continue contribuindo com a maior parcela de consumo até 2040 (IEA, 2019). Além da redução do uso de energia e das emissões de $\mathrm{CO}_{2}$, melhorar a performance energética no setor industrial é uma estratégia vital que minimiza seus custos operacionais (LI; TAO, 2017). Entretanto, a economia de energia não pode ser medida diretamente (EVO, 2016), necessitando de uma abordagem para mensurá-la. A sua modelagem tem implicações no seu processo de medição e na interpretação de seus resultados, sendo considerada como um processo baseado no conhecimento em vez de puramente quantitativa (MICHELI; MARI, 2014). Isto é, presume-se que as propriedades medidas do objeto existem de fato, mas que esses dados coletados resultam de um processo de interpretação, pertencendo a um campo informacional. Ainda, como consequência, o procedimento de medição deve ser definido e o sistema sob medição deve ser projetado e ajustado considerando o contexto e os objetivos para os quais a medição está sendo realizada. Por fim, deve ser dada atenção quando se utiliza qualquer informação em um contexto diferente daquele em que foi pré-concebido, pois isso tem implicações substanciais nos direcionadores, propósitos e usos da medição de desempenho.

Uma forma eficaz para medir o desempenho operacional nas indústrias é encontrado na abordagem Lean, que busca a eficiência da operação, minimizando o uso de recursos, por meio da entrega de valor para o cliente (LEON; CALVO-AMODIO, 2017). O aumento no 
desempenho vem da eliminação do desperdício nas operações (WONG; WONG, 2014). O Mapa de Fluxo de Valor (MFV) é uma ferramenta aplicada (Lacerda et al., 2016; Serrano et al., 2008) para suportar o mapeamento das operações necessárias para agregar valor ao cliente e medir o desempenho atual do sistema analisado (ROTHER; SHOOK, 1999). O MFV tradicional captura dados operacionais de cada etapa de um fluxo produtivo, sendo estendido para coletar outras performances, como energia (CHATTERJEE et al., 2014; JIA et al., 2017; SVENSSON; PARAMONOVA, 2017; HEDLUND e FORCELLINI; 2018), meio ambiente (DARMAWAN; PUTRA; WIGUNA, 2014; NG; LOW; SONG, 2015), sustentabilidade (ISHAK et al., 2017; CHOUDHARY et al., 2019), eletricidade (COSGROVE et al., 2016), água (DAVIES; VAN DER MERWE, 2016), e não se restringindo ao setor industrial, mas como serviços de saúde (L'HOMMEDIEU; KAPPELER, 2010; COSTA et al., 2017), agricultura (FOLINAS et al., 2014; BARTH; MELIN; 2018), construção (ROSENBAUM; TOLEDO; GONZÁLEZ, 2013; GUNDUZ; NASER, 2017), entre outros (SCHMIDTKE; HEISER; HINRICHSEN, 2014; SUNK et al., 2017; XIE et al., 2017).

Apesar da miríade de aplicações do MFV, ele apresenta deficiências na modelagem dos processos independente da variável incorporada. Quando comparado com outras ferramentas de mapeamento, as limitações surgem no que diz respeito ao alcance do escopo, quantificação de dados para análise de desempenho e detalhes do processo (RYBICKA et al., 2015). A comparação de métricas de outras organizações não pode ser usada para fins de benchmarking, isto porque para que organizações utilizem o benchmarking comparando suas operações internamente ou com outras organizações, adotam-se como base um sistema de medição de desempenho que determinará comparativamente a sua direção (PARIDA et al., 2015). Além disso, não são destacados os processos de aquisição dos dados para se chegar ao desempenho energético. Em geral, adiciona-se apenas uma linha indicando o consumo de energia ou outro indicador por produção da operação, sem descrever o processo de medição de desempenho, nem detalhes das partes que integram cada operação ou os recursos energéticos utilizados (HEDLUND; FORCELLINI; 2018). Portanto, este estudo tem o propósito de elaborar uma proposta para um processo de medição do desempenho energético em mapas de fluxo de valor de modo a auxiliar a direcionar os esforços do setor industrial, incluindo a sua correta utilização para fins de benchmarking.

Este estudo está dividido em apresentação dos métodos utilizados para a proposta de medição de desempenho, seguida pelos resultados e discussão. Por fim, apresenta-se a sua conclusão. 


\section{METODOLOGIA}

Para elaborar a proposta de um processo de medição do desempenho energético para Mapas de Fluxo de Valor foi utilizada uma abordagem de pesquisa exploratória. Foram identificadas as principais contribuições na área de conhecimento de medição de desempenho por meio de pesquisas literárias, principalmente em normas e padrões internacionais utilizados na avaliação de projetos de eficiência energética e conservação de energia. Entre as normas e padrões identificados foi escolhida como referência para este estudo a norma internacional ISO 50.006:2016 - Medição do desempenho energético utilizando linhas de base energética (LBE) e indicadores de desempenho energético (IDE) (ISO, 2016), devido a sua aceitação e padronização com atuação global. Como forma de validar os resultados, os itens do processo de proposto serão avaliados e identificados sob a perspectiva do Protocolo Internacional de Medição e Verificação (EVO, 2016).

Como o Mapa de Fluxo de Valor é baseado em quatro etapas, sendo seleção de uma família de produto, mapeamento da situação atual, mapeamento da situação futura e planejamento do trabalho e implementação (ROTHER; SHOOK, 1999), os itens da medição do desempenho sugeridos foram agrupados nas etapas mais apropriadas. Por fim os tópicos da norma utilizada aderentes ao Mapa de Fluxo de Valor, isto é, que podem ser aplicados na ferramenta, foram propostos num sistema de medição de desempenho.

\section{RESULTADOS E DISCUSSÃO}

Com base no método proposto, os resultados são apresentados a seguir. A Tabela 1 relaciona as atividades do processo para medição do desempenho energético com as etapas do desenvolvimento do Mapa de Fluxo de Valor. Os detalhes são apresentados na sequência.

TABELA 1 - Atividades do processo para medição do desempenho energético.

\begin{tabular}{|c|c|}
\hline $\begin{array}{l}\text { Etapa do Mapa de Fluxo de } \\
\text { Valor }\end{array}$ & Atividade do Processo de Medição do Desempenho Energético \\
\hline $\begin{array}{l}\text { Seleção de uma família de } \\
\text { produto }\end{array}$ & $\begin{array}{l}\text { 1. Definir limites dos IDEs } \\
\text { 2. Definir e quantificar os fluxos de energia } \\
\text { 3. Definir e quantificar variáveis relevantes } \\
\text { 4. Definir e quantificar fatores estáticos }\end{array}$ \\
\hline Mapa da situação atual & $\begin{array}{l}\text { 5. Reunião dos dados preliminares } \\
\text { 6. Medição das variáveis energéticas }\end{array}$ \\
\hline
\end{tabular}




\begin{tabular}{|l|l|}
\hline & $\begin{array}{l}\text { 7. Identificação dos IDES conforme seus usuários } \\
8 . \quad \text { Estabelecimento da LBE }\end{array}$ \\
\hline Mapa da situação futura & $\begin{array}{l}\text { Nesta etapa devem ser aplicadas análises de eficiência energética e } \\
\text { conservação de energia, fora do escopo desse estudo. }\end{array}$ \\
\hline $\begin{array}{l}\text { Planejamento do trabalho e } \\
\text { implementação }\end{array}$ & $\begin{array}{l}\text { Repetir as atividades } 1 \text { a } 8 \text { para estabelecer os valores do período de } \\
\text { reporte }\end{array}$ \\
\hline
\end{tabular}

A etapa inicial do Mapa de Fluxo de Valor é a seleção de uma família de produto. Esta etapa tem como objetivo o foco num grupo de produtos que compartilha determinados processos no fluxo de produção (ROTHER; SHOOK, 1999). Para mensurar o desempenho energético é necessário estabelecer quais são os limites da medição, podendo ter diferentes níveis de fronteiras, desde um processo individual, um sistema ou a organização inteira (ISO, 2016). Para o caso do MFV tradicional os processos são delimitados pelos pontos de estoque (ROTHER; SHOOK, 1999) e, assim, a fronteira mais indicada seria a de um processo individual, ou seja, mapear os equipamentos que estão inclusos nessa etapa do processo de produção. Em seguida, identificam-se quais são os insumos energéticos que estão presentes nesse processo produtivo (ISO, 2016). Em outras referências, verifica-se que esta primeira etapa é definida como fronteira de medição, sugerindo a adoção de um perímetro em torno do objeto a ser medido, incluindo todas as formas de energia dentro desses limites (EVO, 2016).

Variáveis relevantes e fatores estáticos devem ser definidos e quantificados, visto que afetam o desempenho energético e tem papel importante quando se faze necessário realizar ajustes na medição (EVO, 2016). As variáveis relevantes impactam diretamente o desempenho energético e podem mudar de forma constante, como por exemplo, parâmetros de produção, horas de trabalho, temperatura, entre outras (ISO, 2016). Já os fatores estáticos são fatores que impactam no desempenho, porém não mudam rotineiramente, como por exemplo, número de operadores, turnos de produção, tamanho do processo (ISO, 2016). A Figura 1 apresenta as informações que devem ser coletadas não sendo necessário excluir aquelas capturadas no mapeamento tradicional. A Figura 2 apresenta um exemplo de dados preliminares coletados para um processo de manutenção de temperatura de alumínio de uma fundição, sendo que de forma ilustrativa os dados de desempenho operacional do MFV tradicional foram retirados para dar ênfase ao desempenho energético. Como é possível observar, com a discriminação do que 
pertence ao processo, incluindo variáveis que o afetam direta ou indiretamente, é possível realizar análises holísticas do comportamento energético.

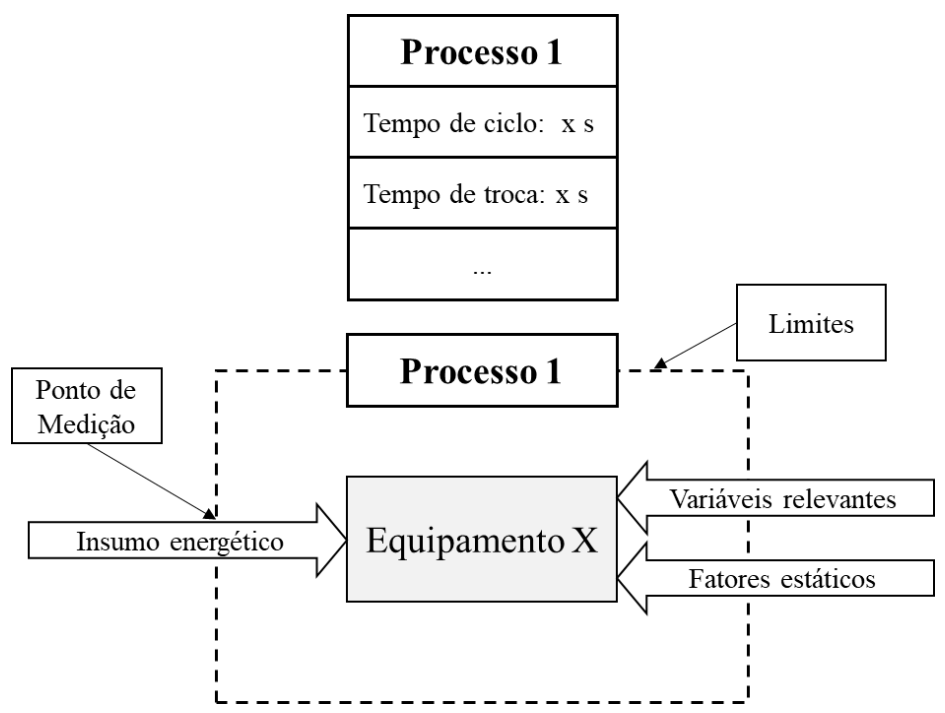

FIGURA 1 - Modelo do processo de medição do desempenho energético para o Mapa de Fluxo de Valor.

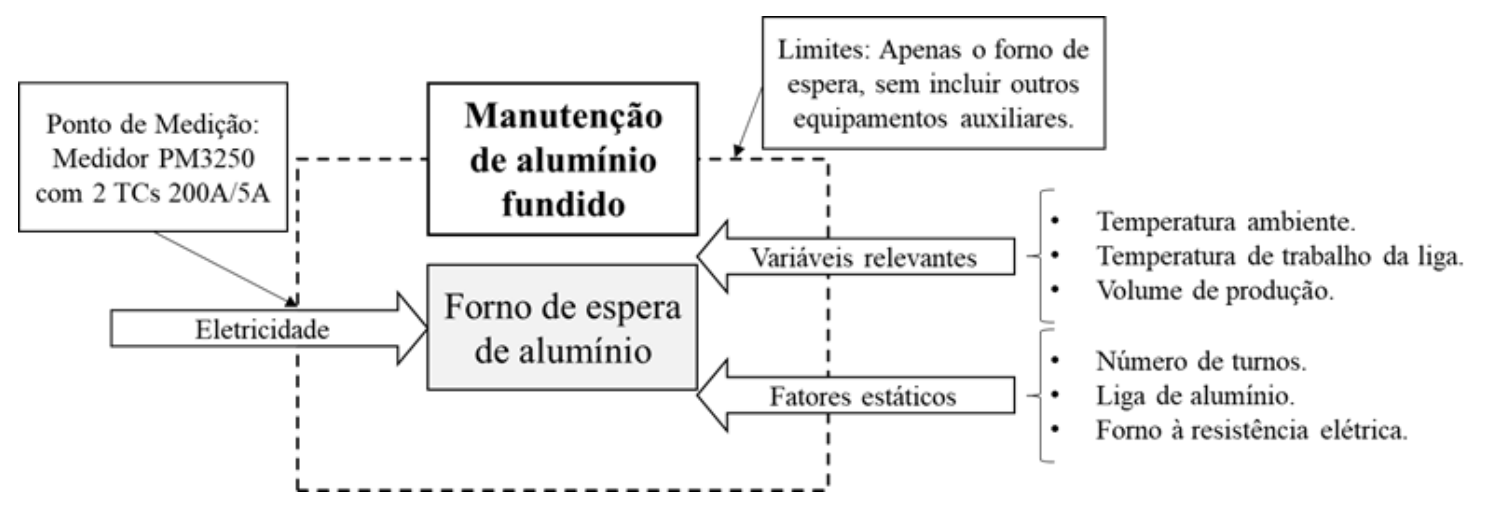

FIGURA 2 - Exemplo de dados preliminares coletados relativos a medição do desempenho energético.

Após a reunião dos dados preliminares, devem-se realizar as medições de forma direta, com o uso de medidores apropriados para cada insumo energético, ou de forma indireta, como a medição de uma variável que afeta o consumo ou por meio de estimativas (ISO, 2016). Recomenda-se que o consumo de energia seja realizado utilizando-se dados ao longo de um período considerável, isto é, as medições devem ocorrer para ciclos completos de operação em condições normais (EVO, 2016)..Para aumentar o nível de confiança nas medições sugere-se a utilização de abordagens estatísticas. Com o auxílio das Equações 1 e 2 (EVO, 2018), é possível determinar o número de amostras, n, para uma variável que apresente distribuição normal. 


$$
n_{0}=\left(\frac{t \times C V}{e_{r e l}}\right)^{2}
$$

EQUAÇÃO 1 - Cálculo para número de amostra inicial.

Onde:

$n_{0}:$ Número de amostra inicial

CV: Coeficiente de variação, relaciona o desvio padrão pela média, utilizar 0,5 quando não se conhece o valor real

$e_{r e l}:$ Nível desejado da precisão relativa

t: Valor de $\mathrm{t}$ de student para um determinado nível de confiança

Se toda a população amostrada não for superior a 20 vezes o tamanho da amostra é possível reduzir o número de amostras por meio da Equação 2.

$$
n=\frac{n_{0} \times N}{n_{0}+N}
$$

EQUAÇÃO 2 - Cálculo ajustado do número de amostras.

Onde:

n: Número de amostra ajustado

N: Número da população.

Por exemplo, para uma confiança de $90 \%, 10 \%$ de precisão relativa e coeficiente de variação de 0,5 , com uma população de 200 medições, teremos um t de student de 1,645, resultando num tamanho de amostra de 67,7. Como a população é três vezes maior que o número de amostras podemos ajustar o tamanho, resultando em 50 amostras para atender os critérios de $90 \%$ de confiança e $\pm 10 \%$ de precisão.

Aliada a minimização de erros de amostras, para reduzir a incerteza nos dados medidos, convém definir uma frequência de coleta de dados, seja horária, diária, semanal etc. (ISO, 2016). Com essas informações, realiza-se uma análise crítica para garantir que os dados representam a realidade do processo medido, podendo pontos discrepantes serem excluídos, desde que se verifique o impacto disso.

A identificação dos Indicadores de Desempenho Energético é realizada com base nos usuários dessas informações, sendo considerada um fator chave (ISO, 2016). Esses podem ser categorizados em valores medidos, proporções de valores medidos, modelos estatísticos ou 
modelos baseados em simuladores de engenharia. Assim, uma medição pode ser utilizada para determinados usuários na forma de valor medido, como para outro grupo na forma de proporção. Exemplos de IDEs (ISO, 2016) são apresentados na Tabela 2.

TABELA 2 - Tipos e métricas de exemplo para os Indicadores de Desempenho Energético (IDEs).

\begin{tabular}{|c|l|}
\hline Tipo de Indicador & \\
\hline \multirow{3}{*}{ Valor medido } & $\begin{array}{l}\text { Consumo de energia, em } \mathrm{kWh} \\
\text { Demanda de energia, em } \mathrm{kW} \\
\text { Consumo de gás, em m }{ }^{3} \\
\text { Consumo de água, em } \mathrm{L}\end{array}$ \\
\hline Proporções & $\begin{array}{l}\text { Consumo específico de eletricidade, em } \mathrm{kWh} / \text { produtos } \\
\text { Custo específico da água, } \mathrm{R} \$ / \mathrm{L}\end{array}$ \\
\hline Modelos estatísticos & Consumo específico de um subsistema, $\mathrm{kWh} / \mathrm{m}^{3}$ \\
\hline Modelos de engenharia & Modelo do consumo de um subsistema com suas variáveis relevantes e estáticas \\
\hline
\end{tabular}

Com as definições anteriores estabelece-se a Linha de Base Energética (LBE) que servirá como referência para comparação do desempenho energético (ISO, 2016). É importante definir o período de dados a ser utilizado para a LBE capturando variações nas variáveis relevantes, como sazonalidades de produção ou padrões climáticos, por exemplo. Quando modelos são utilizados convém a utilização de testes estatísticos para verificar a sua validade. Se não existem mudanças significativas nas variáveis relevantes é possível realizar uma comparação direta entre os períodos de base e após a implementação da melhoria, também chamado de período de reporte. Caso contrário, deve-se realizar uma normalização dos dados, isto é, os dados devem ser ajustados para que sejam considerados sob condições equivalentes (ISO, 2016). 


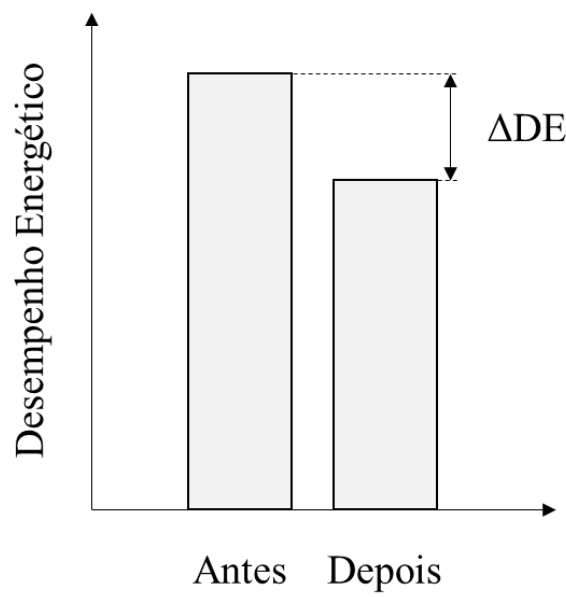

FIGURA 3 - Desempenho energético resultante das medições antes e após a implementação das melhorias no sistema

Após a implementação da melhoria do processo deve-se repetir os passos para reunião dos dados preliminares até a atividade de estabelecer as linhas de base, as quais passarão a ser os dados do período de reporte. A Figura 3 apresenta o conceito da aplicação das linhas de base e de reporte energéticas que representam os dados antes e após a implementação das melhorias. É importante repetir todos os passos, pois durante a melhoria de desempenho operacional de um processo, visando melhorar a eficiência do ponto de vista do Lean é possível que trade-offs ocorram (CAMPOS; VAZQUEZ-BRUST, 2016), isto é, trocas que melhoram a eficiência operacional, mas prejudicam a eficiência energética.

\section{CONCLUSÃO}

Apesar do Mapa de Fluxo de Valor ser uma ferramenta amplamente utilizada não somente para capturar o desempenho operacional e tão pouco relativas apenas a processos industriais, a medição de desempenho não recebe atenção. A falta de um processo mínimo descrevendo as etapas a serem seguidas na coleta de dados afeta não somente o benchmarking de forma interna como na comparação com outras organizações. Assim, verifica-se que há potencial para melhorar o processo de medição de desempenho não somente referente aos insumos energéticos, mas como das outras variáveis de interesse.

Este estudo teve como objetivo a elaboração de um processo para medição do desempenho energético, utilizando como referência a principal norma internacional para medição de performance energética, ISO 50006, associado ao Mapa de Fluxo de Valor estendido a insumos de energia. Ele não se restringe a eletricidade, sendo sugerida sua adoção para qualquer forma 
de energia. Esta proposta procura auxiliar na condução de projetos de melhoria de eficiência energética e conservação de energia em especial no suporte as tomadas de decisão. Ainda, na medida que o processo de medição se inicia acaba gerando uma base de conhecimento e informação para suportar o ciclo da melhoria contínua. Inclusive podendo ser integrado a outras ferramentas de gerenciamento de documentos e conhecimento.

O estudo está limitado as referências internacionais ISO 50.001 e International Performance Measurement and Verification Protocol utilizadas para medição de desempenho energético, sendo necessário aumentar a base de pesquisa para incluir outras referências, incluindo referências acadêmicas. Assim, como sugestão para trabalhos futuros a proposta será expandida para incorporar outras normas, padrões ou artigos científicos, bem como a aplicação da proposta de forma a validar e aumentar a discussão sobre o tema.

\section{REFERÊNCIAS}

BARTH, H., MELIN, M. A Green Lean approach to global competition and climate change in the agricultural sector-A Swedish case study. Journal of Cleaner Production, 204, 183-192., 2018.

CAMPOS, L. M., VAZQUEZ-BRUST, D. A. Lean and green synergies in supply chain management. Supply Chain Management: An International Journal, 21(5), 627-641, 2016.

CHATTERJEE, R., SHARMA, V., MUKHERJEE, S., KUMAR, S. Value stream mapping based on energy and cost system for biodiesel production. International Journal of Sustainable Manufacturing, 3(2), 95-115, 2014.

CHOUDHARY, S., NAYAK, R., DORA, M., MISHRA, N., \& GHADGE, A. SI-TBL: an integrated lean and green approach for improving sustainability performance: a case study of a packaging manufacturing SME in the UK. Production Planning \& Control, 1-16, 2019.

COSGROVE, J., RIVAS DUARTE, M. J., LITTLEWOOD, J., WILGEROTH, P. An energy mapping methodology to reduce energy consumption in manufacturing operations. Proceedings of the Institution of Mechanical Engineers, Part B: Journal of Engineering Manufacture, 232(10), 1731-1740, 2018.

COSTA, L. B. M., FILHO, M. G., RENTES, A. F., BERTANI, T. M., MARDEGAN, R. (2017). Lean healthcare in developing countries: evidence from Brazilian hospitals. The International journal of health planning and management, 32(1), e99-e120, 2017.

DAVIES, E., \& VAN DER MERWE, K. R. Methodology to produce a water and energy stream map (WESM) in the South African manufacturing industry. South African Journal of Industrial Engineering, 27(3), 219-229., 2016

DARMAWAN, M. A., PUTRA, M. P. I. F., \& WIGUNA, B. Value chain analysis for green productivity improvement in the natural rubber supply chain: a case study. Journal of Cleaner Production, 85, 201-211., 2014

EFFICIENCY VALUATION ORGANISATION (EVO). EVO 10.000. International performance measurement and verification protocol. Core Concepts, 2016.

FOLINAS, D., AIDONIS, D., MALINDRETOS, G., VOULGARAKIS, N., TRIANTAFILLOU, D. Greening the agrifood supply chain with lean thinking practices. International Journal of Agricultural Resources, Governance and Ecology, 6, 10(2), 129-145., 2014.

GUNDUZ, M., NASER, A. F. (2017). Cost based value stream mapping as a sustainable construction tool for underground pipeline construction projects. Sustainability, 9(12), 2184., 2017. 
HEDLUND, F. N., FORCELLINI, F. A. Mapa de fluxo de valor estendido a avaliação do desempenho energético em consumidores industriais: um estudo de caso brasileiro. Revista Produção Online, 18(2), 691-712., 2018.

INTERNATIONAL ENERGY AGENCY (IEA). Global Energy \& CO2 Status Report: The latest trends in energy and emissions in 2018, 2019.

INTERNATIONAL ORGANIZATION FOR STANDARDIZATION (ISO). ISO 50.001. Energy Management Systems Standard - Requirements with guidance for use, 2011.

INTERNATIONAL ORGANIZATION FOR STANDARDIZATION (ISO). ISO 50.006, Energy Management Systems Standard - Measuring energy performance using energy baselines (EnB) and energy performance indicators (EnPI) - General principles and guidance, 2014.

ISHAK, A., MOHAMAD, E., SUKARMA, L., MAHMOOD, A. R., RAHMAN, M. A., YAHYA, S. H., SULAIMAN, M. A. (2017). Cleaner Production Implementation using Extended Value Stream Mapping for Enhancing the Sustainability of Lean Manufacturing. Journal of Advanced Manufacturing Technology, 11(1 (1)), 47-60., 2017.

JIA, S., YUAN, Q., LV, J., LIU, Y., REN, D., ZHANG, Z. Therblig-embedded value stream mapping method for lean energy machining. Energy, 138, 1081-1098., 2017.

LAWRENCE, A; NEHLER, T; ANDERSSON, E; KARLSSON, M; THOLLANDER, P. Drivers, Barriers and Success Factors for Energy Management in the Swedish Pulp and Paper Industry. Journal of Cleaner Production, 2019.

LEON, H. C. M.; CALVO-AMODIO, J. Towards lean for sustainability: Understanding the interrelationships between lean and sustainability from a systems thinking perspective. Journal of Cleaner Production, v. 142, p. 4384-4402, 2017.

L'Hommedieu, T., \& Kappeler, K. Lean methodology in iv medication processes in a children's hospital. American Journal of Health-System Pharmacy, 67(24), 2115-2118., 2010.

MICHELI, P; MARI, L. The theory and practice of performance measurement. Management accounting research, 25.2 (2014): 147-156., 2014.

NG, R., LOW, J. S. C., SONG, B. Integrating and implementing Lean and Green practices based on proposition of Carbon-Value Efficiency metric. Journal of Cleaner Production, 95, 242-255. 2015.

PARIDA, A., KUMAR, U., GALAR, D., STENSTRÖM, C. Performance measurement and management for maintenance: a literature review. Journal of Quality in Maintenance Engineering, 21(1), 2-33., 2015.

PEŠALJ, B. ; PAVLOV, A. ; MICHELI, P. The use of management control and performance measurement systems in SMEs: A levers of control perspective. International Journal of Operations \& Production Management, 38(11), 2169-2191., 2018

ROTHER, M.; SHOOK, J. Learning to see. Lean Enterprise Institute, Cambridge, MA, 122 p., 1999.

ROSENBAUM, S., TOLEDO, M., GONZÁLEZ, V. Improving environmental and production performance in construction projects using value-stream mapping: Case study. Journal of Construction Engineering and Management, 140(2), 2013.

RYBICKA, J., TIWARI, A., DEL CAMPO, P. A., HOWARTH, J. Capturing composites manufacturing waste flows through process mapping. Journal of cleaner production, 91, 251-261., 2015.

SCHMIDTKE, D., HEISER, U., HINRICHSEN, O. A simulation-enhanced value stream mapping approach for optimisation of complex production environments. International Journal of Production Research, 52(20), 6146-6160., 2014.

SUNK, A., KUHLANG, P., EDTMAYR, T., SIHN, W. Developments of traditional value stream mapping to enhance personal and organisational system and methods competencies. International Journal of Production Research, 55(13), 3732-3746., 2017.

SVENSSON, A., PARAMONOVA, S. An analytical model for identifying and addressing energy efficiency improvement opportunities in industrial production systems-Model development and testing experiences from Sweden. Journal of cleaner production, 142, 2407-2422., 2017. 
WONG, W. P.; WONG, K. Y. Synergizing an ecosphere of lean for sustainable operations. Journal of cleaner production, v. 85, p. 51-66, 2014.

XIE, H., CHOWDHURY, M. M., ISSA, R. R., \& SHI, W. Simulation of Dynamic Energy Consumption in Modular Construction Manufacturing Processes. Journal of Architectural Engineering, 24(1), 04017034, 2017. 